\title{
USO DE RASTREADORES PARA A IDENTIFICAÇÃO DE EVENTOS ADVERSOS A MEDICAMENTOS*
}

\author{
Cleni Veroneze ${ }^{1}$, Eliane Mara Cesário Pereira Maluf², Fabíola Giordani ${ }^{3}$
}

\begin{abstract}
RESUMO: É um desafio verificar se as estratégias voltadas para aumento da segurança no uso dos medicamentos são efetivas. Dessa maneira, mostra-se clara a necessidade de mecanismos para acompanhar estes resultados. A ferramenta "Global Trigger Tool for Measuring Adverse Events" do Institute for Healthcare Improvement foi desenvolvida para vencer este desafio. O uso de rastreadores para seleção de prontuários, com maior probabilidade de apresentarem eventos adversos, tem sido um método adotado por várias instituições de saúde, em vários países. O objetivo deste relato é descrever a experiência obtida na aplicação do módulo "Medicamento" desta ferramenta em um hospital público brasileiro no estado do Paraná. Realizada no período de novembro de 2012 a janeiro de 2015. A aplicação do método foi de baixo custo e viável para aplicação regular na Instituição.
\end{abstract}

DESCRITORES: Medicamento; Programas de rastreamento; Segurança do paciente; Farmacovigilância.

\section{THE USE OF TRIGGER TOOLSIN THE IDENTIFICATION OF ADVERSE DRUG EVENTS}

ABSTRACT: Checking whether the strategies aimed to improve the safety of drug use are effective is a major challenge. Therefore, the use of mechanisms to monitor these results is clearly necessary. The tool "Global Trigger Tool for Measuring Adverse Events" of the Institute for Healthcare Improvement has been developed to meet this challenge. The use of triggers in the selection of medical records with higher probability of showing adverse drug events (ADE) is adopted by health organizations in several countries. The present experience report aimed to describe the experience of application of the "Medication" module of this tool in a Brazilian public hospital in the state of Paraná. The experience was conducted from November 2012 to January 2015. It is a low-cost method that could be routinely used in the hospital.

DESCRIPTORS: Drug; Trigger tool programs; Patient safety; Pharmacovigilance.

\section{USO DE RASTREADORES PARA IDENTIFICACIÓN DE EVENTOS ADVERSOS A MEDICAMENTOS}

RESUMEN: Verificar la efectividad de las estrategias para mejorar la seguridad en el uso de los medicamentos es un gran desafío. Así, hay una evidente necesidad de establecer modos de acompañar eses resultados. La herramienta "Global Trigger Tool for Measuring Adverse Events" del Institute for Healthcare Improvement fue desarrollada para vencer ese desafío. El uso de rastreadores para selección de prontuarios, con más probabilidad de presentar eventos adversos, viene siendo un método utilizado por variadas instituciones de salud, en distintos países. El objetivo de este relato es describir la experiencia obtenida en la aplicación del módulo "Medicamento" de esta herramienta en un hospital público brasileño en el estado de Paraná. El estudio fue realizado en el periodo de noviembre de 2012 a enero de 2015. La aplicación del método fue de bajo custo y viable para uso regular en la Institución.

DESCRIPTORES: Medicamento; Programas de rastreamento; Seguridad del paciente; "Farmacovigilancia".

*Artigo extraído da dissertação intitulada: "Frequência de eventos adversos a medicamentos em um hospital universitário". Universidade Federal do Paraná, 2015.

${ }^{1}$ Farmacêutica. Mestre em Medicina Interna e Ciências da Saúde. Farmacêutica do Hospital de Clínicas da Universidade Federal do Paraná. Curitiba, PR, Brasil.

${ }^{2}$ Médica. Doutora em Medicina Interna. Docente da Universidade Federal do Paraná. Curitiba, PR, Brasil.

${ }^{3}$ Farmacêutica. Doutora em Saúde Pública. Docente da Universidade Federal Fluminense. Niterói, RJ, Brasil.

Autor Correspondente:

Recebido: 01/03/2016

Cleni Veroneze

Finalizado: $14 / 03 / 2017$

Universidade Federal do Paraná

Av. Cândido de Abreu, 433 - 80.530-000 - Curitiba, PR, Brasil

E-mail: clenivero@yahoo.com.br 


\section{INTRODUÇÃO}

Os medicamentos são um recurso terapêutico decisivo para diminuir o sofrimento dos doentes, entretanto não é isento de riscos. A mensuração do prejuízo ocasionado pelos medicamentos é objeto de muitos estudos que buscam estratégias custo-efetivas para identificá-los ${ }^{(1)}$.

Entre os mecanismos para conhecimento destas ocorrências, estão desde as estratégias mais simples, como a notificação voluntária ${ }^{(1)}$, até a mais complexa representada pelo monitoramento intensivo dos eventos $^{(2)}$. A dependência de incentivo e motivação para a notificação voluntária e os custos elevados do monitoramento intensivo são os maiores limitantes destas estratégias ${ }^{(2)}$.

Neste contexto, o Institute for Healthcare Improvement (IHI) apresentou um método para identificar eventos adversos utilizando rastreadores, denominado "IHI Global Trigger Tool for Measuring Adverse Events" $^{\prime(3)}$, que visa direcionar a revisão de prontuário e assim aumentar a sensibilidade para a identificação de eventos adversos. Esta ferramenta é composta de vários módulos destinados a determinadas linhas do cuidado e contém todo o detalhamento para a sua aplicação, conceitos adotados, composição da equipe de trabalho e treinamento, critérios para seleção dos prontuários, sistemática de revisão destes e forma de sistematização dos resultados.

No módulo "Medicamento", a estratégia consiste em identificação de resultados alterados de exames laboratoriais, administração de medicamentos específicos e descrição de sintomas de reações adversas, para sinalizar os casos com maior possibilidade de se tratarem de eventos adversos a medicamentos (EAM).

A proposta do IHI foi estabelecer um método prático, barato e viável, que pode ser utilizado retrospectivamente para a mensuração regular de eventos adversos e desta forma avaliar a efetividade das ações dos programas de segurança do paciente.

O objetivo desse relato é descrever a experiência obtida na aplicação do módulo "Medicamento" desta ferramenta em um hospital público brasileiro.

\section{RELATO DE EXPERIÊNCIA}

A experiência ocorreu em hospital universitário, com 450 leitos, público, com atendimento exclusivo ao Sistema Único de Saúde (SUS). O módulo de medicamentos do "IHI Global Trigger Tool for Measuring Adverse Events" (3) foi empregado para a identificação da presença de eventos adversos a medicamentos. A pesquisa foi desenvolvida entre novembro de 2012 a janeiro de 2015, após aprovação do projeto em 30 de outubro de 2012 pelo Comitê de Ética em Seres Humanos, sob parecer n 139695.

Em fase preliminar, a lista original de rastreadores foi adaptada com o objetivo de ajuste dos rastreadores propostos à realidade local. Nesta ocasião foram observados se os exames indicados e os medicamentos listados eram adotados na instituição pesquisada, bem como testados diferentes rastreadores para a composição final da lista (Tabela 1).

Tabela 1 - Lista de rastreadores proposta pelo IHI 2009 e resultado após adaptação local. Curitiba, PR, Brasil, 2012

\begin{tabular}{lcc} 
Código & Rastreadores IHI & Rastreadores após ajuste local \\
\hline M1 & Clostridium difficile positivo nas fezes & Mantido \\
\hline M2 & $\begin{array}{c}\text { Tempo de tromboplastina parcialmente } \\
\text { ativada (TTPA) }>100 \text { segundos }\end{array}$ & Mantido \\
\hline M3 & Razão normalizada internacional $(\mathrm{RNI})>6$ & Mantido \\
\hline M4 & Glicemia $<50 \mathrm{mg} / \mathrm{dL}$ & Mantido \\
\hline M5 & Elevação creatinina sérica $2 \mathrm{x}$ valor normal & Mantido \\
\hline M6 & Fitomenadiona & Mantido \\
\hline M7 & Difenidramina & Mantido \\
\hline \hline
\end{tabular}




\begin{tabular}{lcc}
\hline M8 & Flumazenil & Mantido \\
\hline M9 & Naloxona & Mantido \\
\hline M10 & $\begin{array}{c}\text { Antieméticos: Droperidol, Ondansetrona, } \\
\text { Prometazina, Hidroxizine, Metoclopramida, } \\
\text { Trimetobenzamida ou Proclorperazina }\end{array}$ & $\begin{array}{c}\text { Ajustado: Antieméticos: Metoclopramida, } \\
\text { Ondansetrona ou Prometazina e descrição de } \\
\text { náusea/ vômito }\end{array}$ \\
\hline M11 & Sedação excessiva, hipotensão & Mantido \\
\hline M12 & Suspensão abrupta de medicamento & Mantido \\
\hline D13 & Definição local & $\begin{array}{c}\text { Definido por: Transferência para unidade de terapia } \\
\text { intensiva (UTI) }\end{array}$
\end{tabular}

FONTE: Institute for Healthcare Improvement (IHI) e autor

O relato da experiência foi baseado na revisão de 192 prontuários de pacientes adultos após a alta hospitalar, sendo a execução realizada em três etapas (Figura 1). Na primeira etapa, realizada por acadêmicos de medicina ou farmácia do último ano de formação, foi verificada a presença de rastreador ou de algum evento adverso descrito claramente. A segunda etapa consistiu na avaliação dos casos triados para a constatação da ocorrência do evento adverso a medicamento e foi apoiada pela consulta a literatura disponível em bases de dados de informações sobre medicamentos, como o UpToDate ${ }^{\circledR}$ e Medscape. Na terceira etapa, uma comissão multiprofissional(4), composta por médico, enfermeiro e farmacêutico, avaliou os casos e definiu por meio de consenso se havia ocorrido um evento adverso a medicamento.

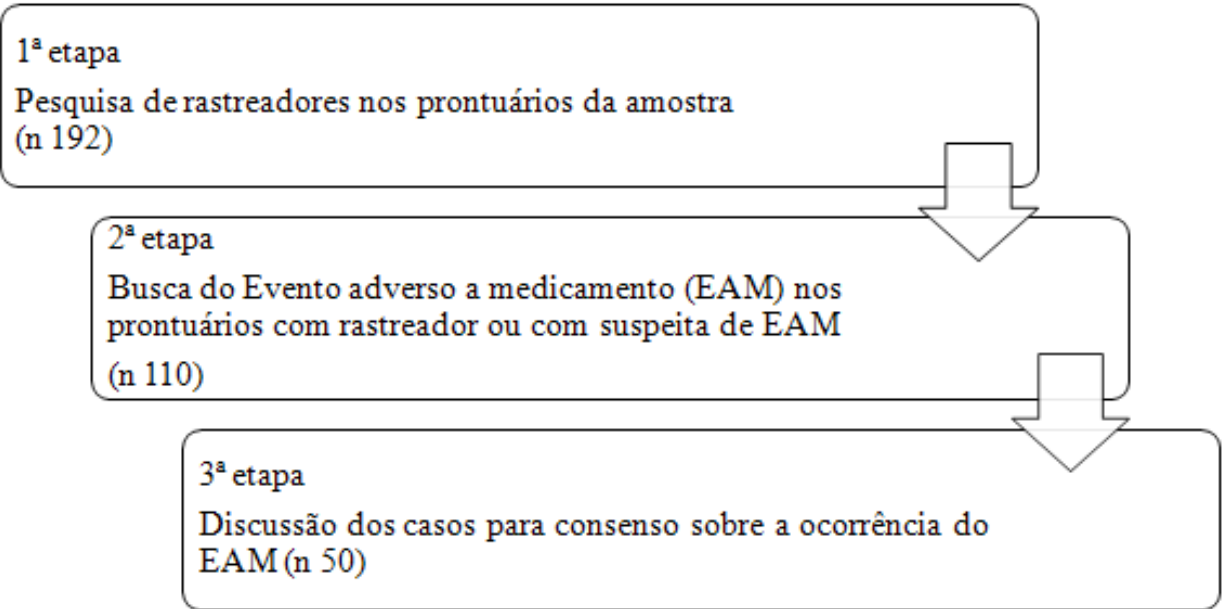

Figura 1 - Descrição do método adaptado e o número de prontuários avaliados em cada etapa. Curitiba, PR, Brasil, 2014

A aplicação do método resultou no diagnóstico situacional da instituição, uma vez que tornou possível conhecer a dimensão do número de eventos adversos a medicamentos ocorridos no período avaliado.

\section{DISCUSSÃO}

O método original proposto pelo IHI(1) recomenda a avaliação dos prontuários com a identificação do rastreador e a verificação do EAM simultaneamente, utilizando um tempo de 20 minutos, sendo a confirmação do EAM realizada posteriormente por um médico ${ }^{(1,3,5)}$.

Nessa pesquisa, a aplicação da ferramenta foi distribuída em três etapas, modificação que favoreceu sua execução sem prejuízos para o resultado. 
O apoio de acadêmicos na primeira etapa, não apenas contribuiu para diminuir o tempo despendido pelo pesquisador nesta fase mais objetiva e menos complexa do método, mas também constituiu um mecanismo para a formação destes futuros profissionais ${ }^{(6-7)}$, aumentando a percepção para a relevância do tema.

A verificação da ocorrência do evento adverso na segunda etapa foi a fase que requereu mais tempo para execução, tendo em vista a necessidade de consulta específica em base de dados para cada um dos medicamentos suspeitos de causar o evento. Esta condição fortaleceu a indicação do caso como evento adverso a medicamento.

A confirmação do EAM realizada por profissionais ${ }^{(4)}$ de formações diferentes, na terceira etapa, propiciou discussão mais aprofundada sobre cada caso, respeitando o conhecimento específico de cada categoria, resultando na conclusão do caso com mais propriedade e no aumento do conhecimento para todos os participantes.

Mesmo com a padronização dos instrumentos de coleta, conceitos e treinamento dos participantes, o tempo gasto para a obtenção dos dados nos casos confirmados de evento adverso a medicamento foi superior em três vezes ao sugerido de 20 minutos pelo método. Este fato pode ser decorrente de algumas características estruturais da instituição. Os prontuários eram manuscritos, apresentavam problemas de legibilidade e uso de abreviações não normatizadas, o que tornou necessária uma análise minuciosa para evitar perdas de dados. O envolvimento emocional com determinados casos também pode ter contribuído para este tempo maior.

Ao final da experiência, foi possível constatar que o método é exequível nesta instituição, o que resultou na sua inserção como uma das estratégias para mensurar regularmente a ocorrência de eventos adversos no local, implementação estimulada pela legislação ${ }^{(8)}$.

\section{CONCLUSÃO}

O método "IHI Global Trigger Tool for Measuring Adverse Events"(3) apresentou-se útil e exequível para a aplicação regular na instituição para conhecimento dos eventos adversos a medicamentos, mesmo com tempo maior que o estabelecido. A implantação de prontuário eletrônico na instituição, além de outros benefícios ${ }^{(9)}$, pode facilitar a aplicação desta metodologia.

Para o futuro, a aplicação dos outros módulos da ferramenta, como cuidado, cirurgia, cuidado intensivo, perinatal e emergência do "IHI Global Trigger Tool for Measuring Adverse Events"(3), para identificação dos eventos adversos relacionados a outros aspectos do cuidado, é uma alternativa interessante para compor as estratégias de mensuração dos incidentes com dano.

É por meio do conhecimento da ocorrência dos eventos adversos e da identificação de quais podem ser evitados que se pode buscar o aprimoramento dos processos de trabalho e assim torná-los mais $\operatorname{seguros}^{(10-12)}$. Em adição e considerando que estratégias combinadas podem aumentar a identificação dos incidentes com dano, o método descrito apresenta-se como recurso de muita utilidade em benefício da segurança do paciente.

\section{REFERÊNCIAS}

1. Institute for Healthcare Improvement (IHI). Trigger Tool for Measuring Adverse Drug Events. [Internet] 2004 [acesso em 26 set 2014]. Disponível: http://www.ihi.org/resources/Pages/Tools/ TriggerToolforMeasuringAdverseDrugEvents.aspx.

2. Dias MF. Introdução à farmacovigilância. In: Storpitis S, Mori ALPM, Yochiy A, Riberio E, Porta V. Ciências farmacêuticas: farmácia clínica e atenção farmacêutica. Rio de Janeiro: Guanabara Koogan; 2008. p.46-63.

3. Griffin FA, Resar RK. IHI Global Trigger Tool for Measuring Adverse Events. $2^{\mathrm{a}}$ ed. IHI Innovation Series white paper. Cambridge, Massachusetts: Institute for Healthcare Improvement; 2009.

4. Giordani F, Rozenfeld S, Martins M. Adverse drug events identified by triggers at a teaching hospital in Brazil. 
BMC Pharmacol Toxicol. [Internet] 2014;(15) [acesso em 20 fev 2016]. Disponível: https://dx.doi.org/10.1186/20506511-15-71.

5. Griffin FA, Resar RK. IHI Global Trigger Tool for Measuring Adverse Events. IHI Innovation Series white paper. Cambridge, Massachusetts: Institute for Healthcare Improvement; 2007.

6. Giordani F, Rozenfeld S, de Oliveira DFM, Versa GLGS, Terencio JS, Caldeira LF, et al. Vigilância de eventos adversos a medicamentos em hospitais: aplicação e desempenho de rastreadores. Rev. bras. epidemiol. [Internet] 2012;15(3) [acesso em 20 fev 2016]. Disponível: https://dx.doi.org/10.1590/S1415-790X2012000300002.

7. Rozenfeld S, Chaves SMC, Reis LGC, Martins M, Travassos C, Mendes W, et al. Efeitos adversos a medicamentos em hospital público: estudo piloto. Rev. Saúde Pública. [Internet] 2009;43(5) [acesso em 20 fev 2016]. Disponível: https://dx.doi.org/10.1590/S0034-89102009005000051.

8. Ministério da Saúde (BR). Agência Nacional de Vigilância Sanitária. Resolução da Diretoria Colegiada - RDC n.o 36, de 25 de julho de 2013. Institui ações para a segurança do paciente em serviços de saúde e dá outras providências. Diário Oficial da União, Brasília, 26 jul. 2013. Seção 1.

9. Pavão ALB, Andrade D, Mendes W, Martins M, Travassos C. Estudo de incidência de eventos adversos hospitalares, Rio de Janeiro, Brasil: avaliação da qualidade do prontuário do paciente. Rev. bras. epidemiol. [Internet] 2011;14(4) [acesso em 12 nov 2016]. Disponível: http://dx.doi.org/10.1590/S1415-790X2011000400012.

10. Ferner RE. An agenda for UK clinical pharmacology: medication errors. Br J Clin Pharmacol. 2012;73(6):912-6. 11. Thomas EJ, Petersen LA. Measuring errors and adverse events in health care. J Gen Intern Med. 2003;18(1):617.

12. Mendes W, Pavão ALB, Martins M, Moura MLO, Travassos C. The feature of preventable adverse events in hospitals in the State of Rio de Janeiro, Brazil. Rev Assoc Med Bras. [Internet] 2013;59(5) [acesso em 11 nov 2016]. Disponível: http://dx.doi.org/10.1016/j.ramb.2013.03.002. 